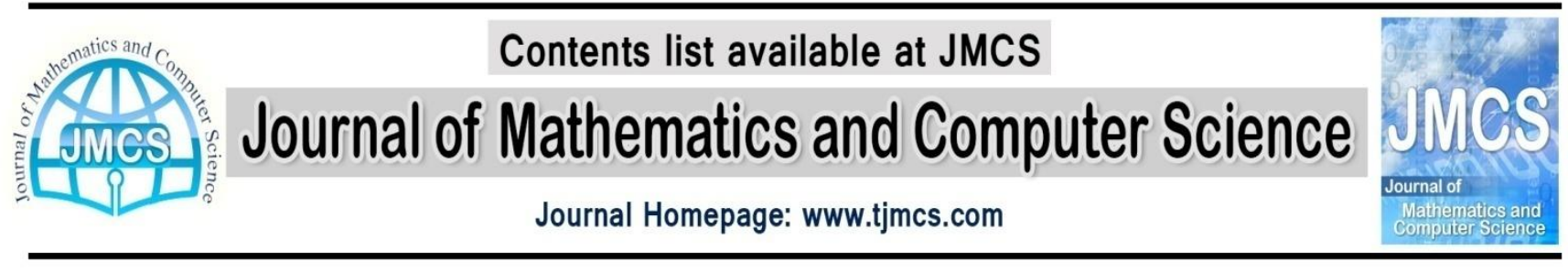

\title{
Applications of OPLS Statistical Method in Medicine
}

\author{
Kianoush Fathi Vajargah*1, ${ }^{*}$ obabe Mehdizadeh $^{2}$, Homayoun Sadeghi-Bazargani $^{3}$ \\ ${ }^{1,2}$ Department of Statistics, Islamic Azad University, North branch, Tehran, Iran \\ ${ }^{3}$ Department of Statistics and Epidemiology, Tabriz University of Medical Sciences, Tabriz, Iran \\ kfathi@iau-tnb.ac.ir , mehdizadeh18@yahoo.com, homayoun.sadeghi@gmail.com
}

\begin{abstract}
Studies related to prognosis in medicine result in a large volume of variables if clinical and laboratory variables are simultaneously accompanied with new imaging techniques; this issue causes problems for classical statistical methods such as logistic and linear regression. Among these cases, emergence of multicollinearity or close linear correlation between regression variables when the number of regression variables is high can be pointed out. Emergence of multicollinearity is inappropriate for ordinary least squares of regression model. PLS is a well-known method for connecting two X and Y data matrices using a multicollinearity model. OPLS is the product of a change which has occurred on PLS method in recent years. Considering application problems of linear regression method, applying an alternative method is a requirement. Using OPLS method can reduce model complexity and develop its power.
\end{abstract}

Key words: Medical studies, linear regression, PLS , OPLS

\section{Introduction}

In studies related to medicine, biology, chemistry, social and behavioral sciences and other sciences, researchers collect data related to several variables. Data related to these variables are called "multivariate data" and multivariate statistical methods are applied for their analysis. In most situations, there are a large number of variables (sometimes more than the number of observations). In such a situation, using multivariate linear regression method is faced with problems due to emergence of multicollinearity (correlation between the variables) because least squares method offers poor estimation of each individual parameter of the model, which may seriously limit application and usage of regression model in inference and prediction. Various methods have been proposed for dealing with the problem of multicollinearity, each of which has specific limitations. So, principal component analysis method was first proposed in 
order to solve this problem. Although this method solves multicollinearity problem, the problem of optimal sub-category selection from among the predictors is still left. Principal component regression analyzes $\mathrm{X}$ in order to obtain components which can explain $\mathrm{X}$ in the best way. In contrast, PLS (partial least squares) regression finds components of $\mathrm{X}$ which can predict $\mathrm{Y}$ in the best way. OPLS (projection to latent structures) is the improved PLS method, which removes $\mathrm{X}$ changes (predictor) that have no correlation with $\mathrm{Y}$ (response variable). In mathematical terms, it eliminates systematic changes in $\mathrm{X}$ matrix which are perpendicular to $\mathrm{Y}$. Therefore, considering the above-mentioned items, it is required for clinicians to have basic familiarity with novel methods of statistical analysis such as PLS and OPLS. In this article, the possibility of using OPLS and PLS models was discussed by considering appropriate practical examples.

\section{Multicollinearity}

Regression models are used in a wide field of application. A serious problem in applying regression model is multicollinearity or close linear correlation between regression variables in case there are many regression variables. Multicollinearity closely depends on regression variables. Close dependence causes difficulty in estimating capability of regression coefficients. Emergence of multicollinearity is inappropriate for ordinary least squares regression analysis. In this situation, variance of estimator of least squares may be highly large and prediction will be inaccurate. On the other hand, the number of observations may be less than that of regression variables; in this situation, there is no invertible matrix and thus no unbiased estimator of unique least squares for the matrix coefficient (1).

Effects of multicollinearity: Although lack of collinearity is not among primary assumptions of regression and does not completely invalidate regression analysis, since it leads to an error in model determination, it can cause difficulties in calculation, interpretation and prediction. Multicollinearity also results in estimating least squares $Q U O T E \hat{\beta}_{j}$ which are very large in terms of absolute value. Generally, when there is severe multicollinearity, least squares method offers poor estimation of each individual parameter of the model, which may seriously limit application of regression model for inference and prediction (1). Methods for Confronting Collinearity Problem:

Re-definition of the model in terms of a set which is smaller than regression variables, conducting preliminary studies which use only subcategories of basic variables, relating $\mathrm{Y}$ response to main components of prediction variables which decide on the regression variables that should be eliminated from the model, collecting additional data which is not always possible unfortunately due to economic bounds or lack of access to studied sampling process, ridged regression and so forth are some examples of methods for confronting collinearity problem. In the first two methods, relationships between regression variables are ignored and can lead to unacceptable results (1). 


\section{Three Alternative Methods for Managing Multiplicity of Variables}

3.1. Principal Component Analysis (PCA): Scientists of biology, physics, behavioral and social sciences, physicians and other people usually collect data related to several variables within their studied topic. In other words, instead of having one variable, they study some other variables. The data related to these variables are called multivariate data and multivariate statistical methods are used for their analysis. These variables are either continuous or discrete; sometimes, some of them are continuous and some others are discrete. For example, a physician studies height, weight, gender and blood pressure of a patient simultaneously in order to investigate a disease. Principal component analysis is one of the main multivariate data analysis methods, the objective of which is to reduce dimension of the studied issue. Using principal components analysis can substitute a number of correlated explanatory variables (independent variables) with a limited number of new uncorrelated explanatory variables $(\mathrm{p}>\mathrm{m})$ which are linear combination of initial variables. These new uncorrelated variables are called "principal components" and their study and investigation are performed as principal components analysis (PCA). Therefore, not only dimension of the issue is reduced, but also multicollinearity does not occur (2).

3.2. Partial least squares regression (PLS): Partial least squares regression is a new method which is a generalization and combination of analysis method of principal components and multicollinearity. PLS is a model for relating two data matrices of $\mathrm{X}$ and $\mathrm{Y}$ by a multivariate linear model $(3,4)$. Its origin dates back to the $60 \mathrm{~s}, 70 \mathrm{~s}$ and 80 s of previous century when Herman Wold $^{1}$ was seriously looking for and interpreting models and methods for social sciences. Father of these algorithms is Herman Wuld (1996) (5). Usefulness of this method is in its ability to analyze data with large errors (noisy), data located on a straight line (collinearity) and even incomplete variables in two $\mathrm{X}$ and $\mathrm{Y}$ matrices. Accuracy of a PLS model for parameters related to observations (samples, combinations, subjects and items) is improved by increasing the number of suitable $X$ variables (6). The objective of partial least squares regression is to predict $\mathrm{Y}$ using $\mathrm{X}$ and describe their common structure. When $\mathrm{Y}$ is a vector and $\mathrm{X}$ is a high-ranking matrix, this objective can be estimated using partial multiple regression. When the number of predictor variables is more than the number of observations, it is not possible to use ordinary regression method due to multicollinearity. Orthogonal principal components solve problem of multicollinearity; however, problem of selecting an optimal subcategory of the predictors is still left because these components have been selected for describing X, instead of Y. Therefore, there is no guarantee for the issue that "the principal components which describe $\mathrm{X}$ depend on Y". 
PLS regression finds some components of $\mathrm{X}$ which predict $\mathrm{Y}$ in the best way (some components of $\mathrm{X}$ which are related to $\mathrm{Y}$ ). In particular, partial least squares regression seeks a set of principal components (called latent ${ }^{2}$ vectors) which simultaneously analyzes $\mathrm{X}$ and $\mathrm{Y}$ with the constraint that these components explain the variance between $\mathrm{X}$ and $\mathrm{Y}$ as much as possible. This stage generalizes analysis of principal components. During one regression stage, $\mathrm{X}$ analysis is followed for $\mathrm{Y}$ prediction (7).

3.3.OPLS: OPLS method which is the improved form of PLS was first presented in 2002. The main objective of OPLS is to separate systematic changes in X into two parts; one of them is in linear relationship with Y and another is irrelevant to Y (perpendicular to it) (8-10). Similar to PCA, PLS and other multivariate methods, the first step in OPLS is preprocessing the input data (centralizing mean and scaling variance) because there may exist certain parameters in the model which are affected by increasing the variables' variance. OPLS provides a way for eliminating systematic changes in the set of $\mathrm{X}$ input data which are not in correlation with set of $\mathrm{Y}$ response data. In other words, some changes in $\mathrm{X}$ which are perpendicular to $\mathrm{Y}$ are eliminated. Uncorrelated changes in $\mathrm{X}$ are separated from correlated ones with this additional advantage that uncorrelated changes can be studied and analyzed separately. Eliminating uncorrelated changes in the data before date modeling not only is interesting in terms of prediction, but also could improve the ability of interpreting results of the models (11).

\section{OPLS Properties}

In semi-empirical modeling, evident advantages of OPLS models include their simplicity compared with PLS method and their easy interpretation since uncorrelated and correlated changes are separated. OPLS offers an improved detection limit for remote data in privileges ${ }^{3}$ because uncorrelated changes in $\mathrm{X}$ can have different statistical distributions from correlated ones. Another advantage of OPLS is that inner repetition is not time-consuming which can accelerate the calculation process. Analyzing correlated changes is always useful. Probably, source of turbulent changes can be distinguished, eliminated or finally realized (12).

A brief description of OPLS

Experience $^{3}$ 


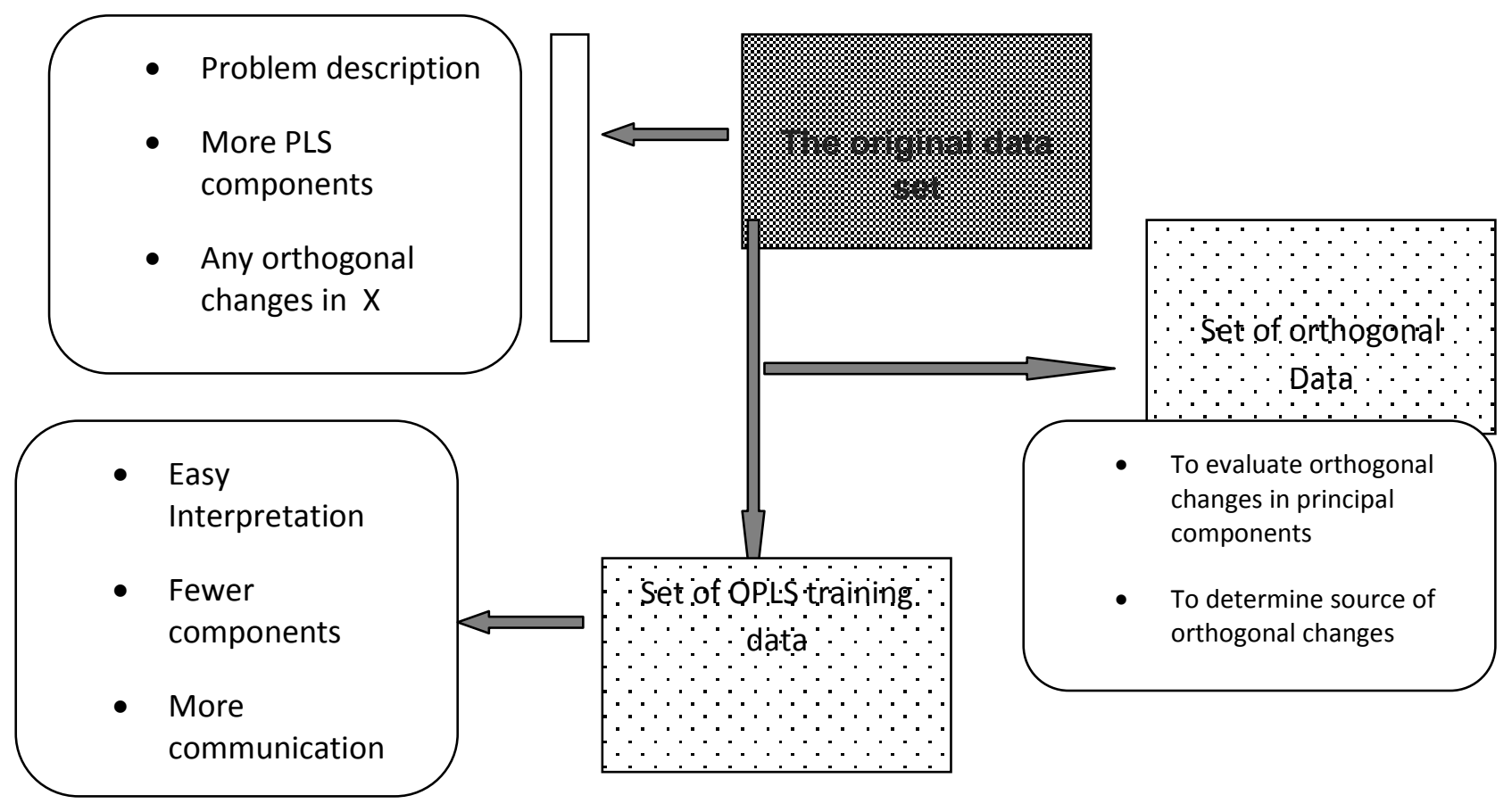

Below, two examples of studies in medical field which have used these methods are mentioned. Both studies have utilized SIMCA (ver. 12) software, which is special software for modeling techniques of supervised models. OPLS is a tool in SIMCA software so that it is accessible based on standards of PLS and PCA models:

\section{The First Sample:}

This study was done to compare new statistical methods of OPLS-DA and PLS regression in management of multiple variables in a case-control study on burns. The data used in this study for implementing and comparing statistical models were drawn from a continuous case-control study for determining the damage induced by unintentional burns. The studied samples were the ones which referred to Burn Centre of Northwest of Iran. The control group included below 14 year old children, who referred to Tabriz Children Hospital. Before the modeling process, the data were divided into two parts. The set of training data consisted of 316 observations and set of predictor data included 80 observations. OPLS-DA which 
was conducted in this study on the data was discussed a bit later than OPLS, namely in 2006, and all the recognized advantages of OPLS modeling were kept in field of audit analysis (13).

Both PLS-Da and OPLS-DA models were fitted on a set of training data consisting of $2 \pm 247$ and 316 observations in both groups. The least possible number of components was 3 components in PLS-DA. But only one predictor component in OPLS-Da model was obtained by extracting two orthogonal components in X. Gaining a predictor component in OPLS as the only component relating to Y made model interpretation easier than PLS model. OPLS-DA was approved owing to its better interpretation as a model superior to PLS-DA. Authors of this study encouraged epidemiologists who study injuries and damage and also statisticians who apply and evaluate these methods in analyzing the data related to damage in order to increase its studying ability and statistical validity. Other epidemiological studies can take advantage of this method using a higher number of dependent variables (14).

\section{The Second Sample:}

This study was a prospective study and its general objective was to determine role of Transcranial Doppler in prognosis of ischemic strokes. The studied sample was a set of data obtained from 116 patients with diagnosis of ischemic stroke who were hospitalized in Neurology Unit, Razi Hospital. During the first week, TCD (Transcranial Doppler) hospitalization was done (for the patients diagnosed with stroke embolic, TCD was done in the first $48 \mathrm{~h}$; in case the first $48 \mathrm{~h}$ was passed, they were mostly removed from the study based on re-establishing of blood flow (Ray Canalization) and, for other cases, the first week was acceptable.) Then, UNSS (Unified Neurological Stroke Scale) questionnaires were completed by another person who was unaware of TCD result. Finally, the same person re-completed the forms at the end of 6 months. UNSS form is a simple form for evaluating clinical status of neurological patients and the objective of its design is to facilitate estimating the patients' disability; it can measure awareness, speech, eye movement, walking, muscle strength and power of organs. Transcranial Doppler is a method for evaluating cervical carotid arteries and intracranial vessels which studies hemodynamic situation of vessels (blood flow velocity and its direction) (15-17).

Four groups of variables were analyzed in this study: the first group: demographic variables and medical records; the second group: laboratory variables; the third group: variables of Transcranial Doppler Sonography (TCD); the forth group: variables of UNSS scale. The obtained UNSS scale was considered a response variable which was predicted by other variables. R2 and Q2 parameters were calculated for each

of the methods and the following results were obtained: considering $\mathrm{R}^{2}$ and $\mathrm{Q}^{2}$ values for PLS model, one component was obtained. One predicted and two orthogonal components were also obtained for OPLS 
model. Values of $\mathrm{R}^{2} \mathrm{Y}$ and $\mathrm{Q}^{2}$ for PLS model were calculated as 0.53 and 0.14 , respectively. $\mathrm{R}^{2} \mathrm{Y}$ and $\mathrm{Q}^{2}$ for OPLS model were calculated as 0.73 and 0.37 , respectively (Figures 1 and 2).

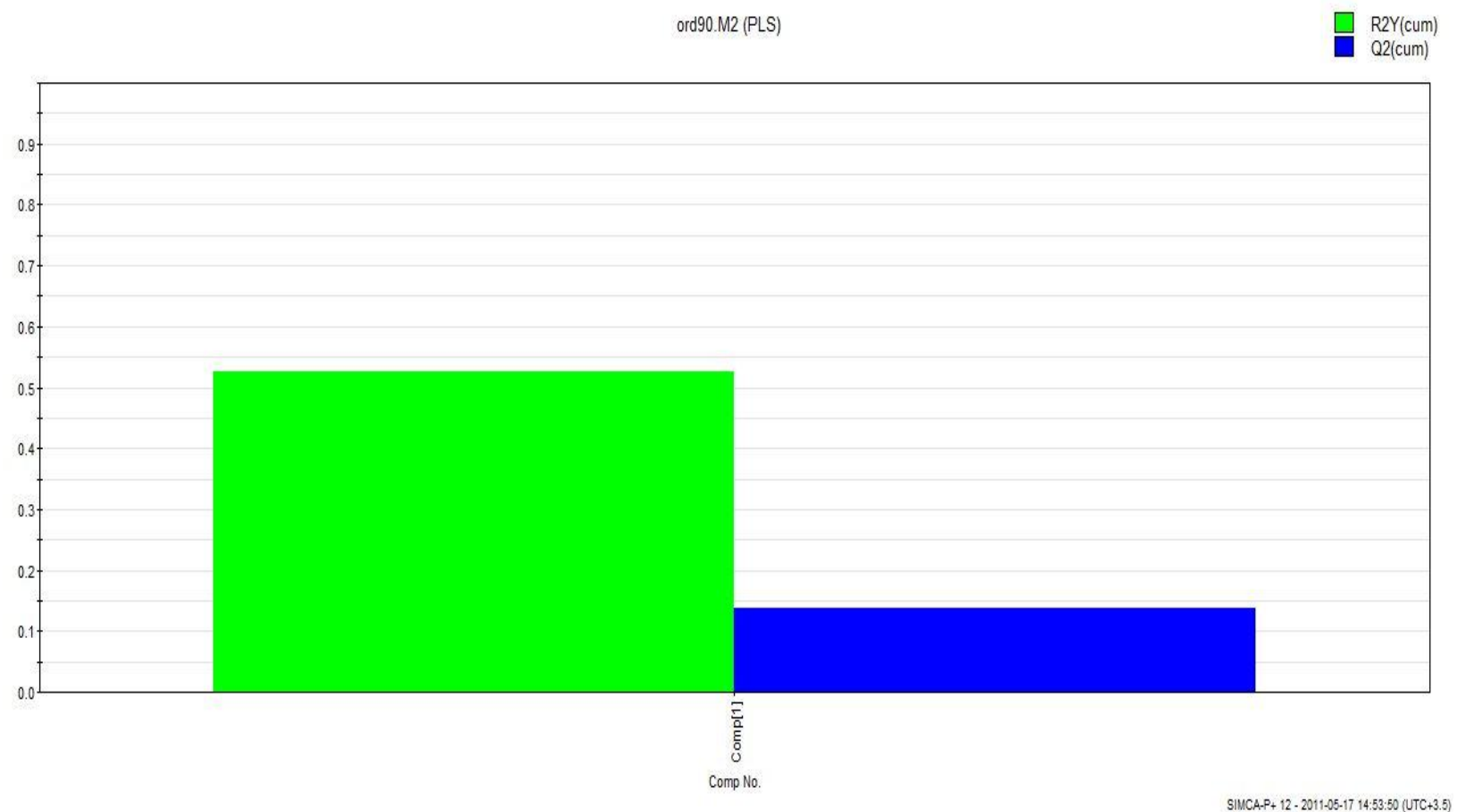

Figure 1: Calculated values of R2 and Q2 for the PLS method

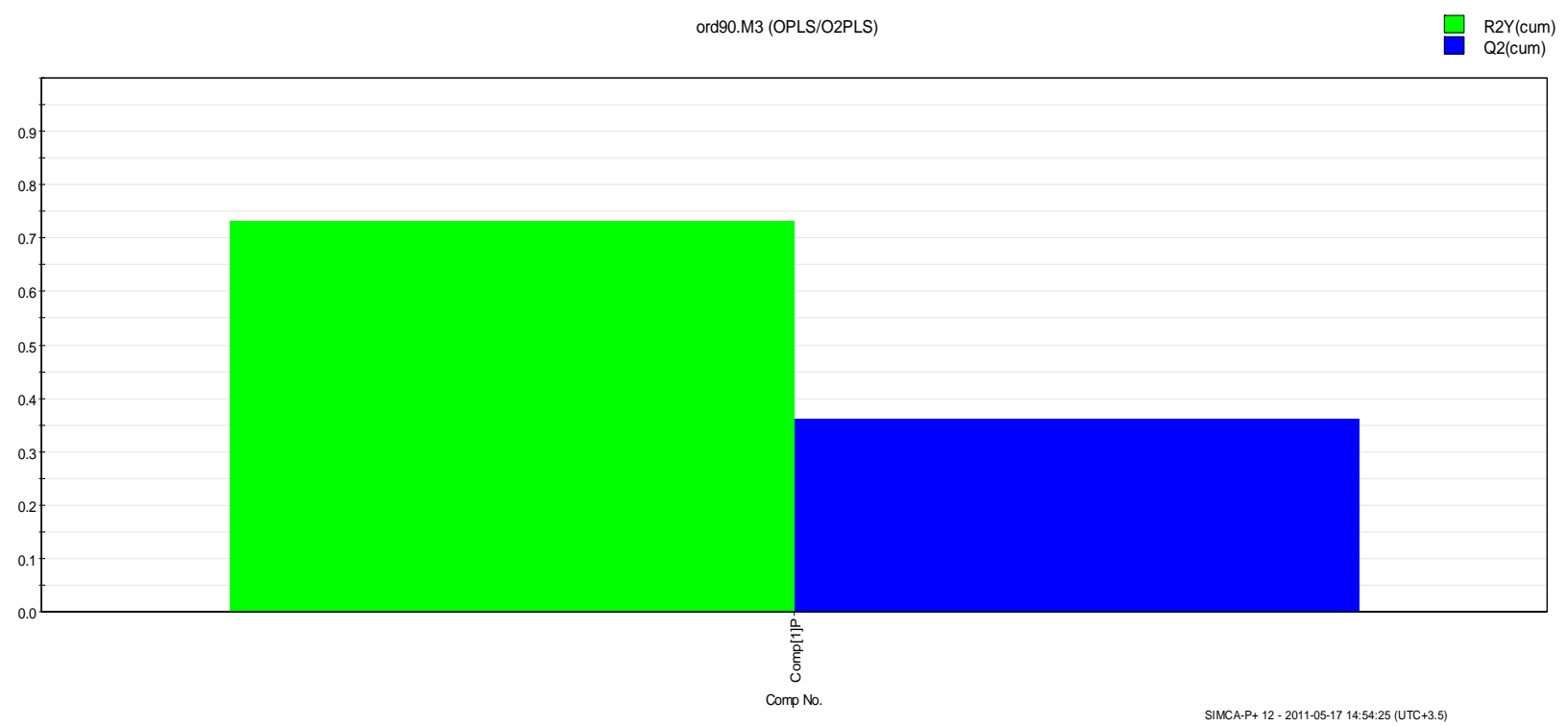

Figure 2: Calculated values of $\mathrm{R}^{2}$ and $\mathrm{Q}^{2}$ for the OPLS method 
By comparing the calculated indices for two models, it was found that OPLS model was superior to PLS model in terms of goodness of fit and ability to predict the model. In this study, based on very high number of variables resulting from Transcranial Doppler Sonography (TCD) in patients with stroke compared to the limited number of studied observations, inclusion of other demographic and laboratory variables in the study and close correlation between TCD variables, OPLS application was suggested as an alternative method for linear regression and both PLS and OPLS were conducted on the data. In the results obtained by both methods of PLS and OPLS, one component and one predictor and two orthogonal components were obtained, respectively. An important property of OPLS is simpler interpretation of results than PLS since OPLS results in only one predictor component and other components are orthogonal. Although PLS only offered one component in this study, in other references, several components have been obtained for PLS method; the more the number of components, the more complicated the result interpretation would be. This study presented similar results for both methods in terms of determining significant variables in other cases; but, OPLS method showed a significantly less number of variables because of removing the variables which had no role in prediction and considering them as orthogonal components (18).

\section{Discussion and Results:}

Although using likelihood estimation and logistic regression methods has some advantages compared to linear regression analysis method, they have some limitations like other regression methods, which include independence of $\mathrm{X}$ variables, accuracy of $\mathrm{X}$ variables and randomization of error distribution. Power and missing data are among other concerns for classical regression model (11). Limitations of these methods require using alternative methods with supplement while being applied in studies which consist of a large number of correlated variables.

The first problem which stems from the large number of variables while using classical regression analysis method (especially, logistic regression) is power requirement. In case the number of variables is more than that of observations, it will be more complicated. Although methods of estimation (maximum conditional and unconditional likelihood) are useful for logistic regression, the correlation between predictor variables which increases by the increase in the number of variables is a problem which should be properly dealt with.

Multiple correlation is also an issue which arises from facing dependant variable (19). Multiple correlation inflates variances of parameters estimation. Generally, when there is severe multicollinearity, method of least squares presents weak estimation of each individual parameters of the model, which may severely limit application and usage of regression model for inference and prediction (1). In order to 
confront the aforesaid problems, principal component analysis (PCA) has been proposed. Orthogonal principal components can solve multicollinearity problem; but, problem of optimal subcategory selection from among the predictors is still left. These components are chosen to explain X, instead of $\mathrm{Y}$. Therefore, there is no guarantee for the point that "Principle components which describe $\mathrm{X}$ depend on $\mathrm{Y}$ ".

A well-known method called PLS regression produces a set of main predictor variables. In latent component, interior variables are correlated with each other (11). Thus, the correlation between predictor variables which seem to be irritating in classical regression methods can be a useful source of information about a group of variables (14). Therefore, PLS regression methods have many advantages over other classic and traditional methods that are based on components. Independence of variables is not PLS's prerequisite. This method does not suffer from multicollinearity and limitation of various variables. This method can also cope with the problems and average number of missing data in both $\mathrm{X}$ and $\mathrm{Y}$ variables. In comparison with classical regression methods in modeling PLS, the statistician will be never forced to extract a set of dummy variables or the calculations which are related to principal variables. For example, a statistician can mention income of a husband and wife together with total income of the couple in a model. But, this case cannot be used in classic regression models because of the concern about being a source of multicollinearity (14).

There are some major issues about application of PLS model: difficulty of interpretation because of the number of components in the model, arbitrary methodology for definitions of number of components, problems due to the need for pre-processing and impact of effective values. Also, most readers are not familiar with the concept of latent variables and inadequate analysis of orthogonal changes of data (11).

Wold et al. were the pioneers in introducing OSC (orthogonal signal correction) for eliminating systematic changes from matrix X which were unrelated (orthogonal) to Y response variable (20). Results of OSC in the improved model could be interpreted; but, the main problem of OSC method is about the concerns for pre-processing risks of OSC components and assuring that prediction power does not significantly change (14).

Another method, as the improved method of PLS, which was later extended was OPLS. The main objective of OPLS is to separate systematic changes in X into two parts; one part is associated with linear relation with $\mathrm{Y}$ and another is unrelated to $\mathrm{Y}$ (perpendicular to it). Similar to PCA, PLS and other multivariate methods, the first step in OPLS is pre-processing input data (centralizing mean and scaling variance) since there may be some parameters in the model which are affected by increased variance of the variables. OPLS provides a way for eliminating systematic changes in a set of $\mathrm{X}$ input data which are not in correlation to $\mathrm{Y}$ response data; i.e. eliminating some changes in $\mathrm{X}$ which are perpendicular to $\mathrm{Y}$. 
The uncorrelated changes in $\mathrm{X}$ are separated from the correlated ones assuming that these changes can be studied and analyzed separately. Removing uncorrelated changes of data before date modeling not only is interesting in terms of prediction but also could improve the interpretation ability of model results (11). At semi-empirical modeling level, obvious advantages of OPLS models are their simplicity compared with PLS and easy interpretation because uncorrelated and correlated changes are separated. OPLS gives an improved detection limit for remote data in privileges since uncorrelated changes in $\mathrm{X}$ can have different statistical distribution from the correlated ones. Another advantage of OPLS is that its presented inner repetition ${ }^{4}$ is not time-consuming, which could accelerate calculation process (18).

The present authors recommend epidemiology experts as well as statisticians to apply and study this model's application in various fields of medicine in order to broaden statistical power and validity of studies. Other epidemiologic studies which deal with a large number of correlated variables can also take advantage of this method. Since this subject is new and there are a limited number of studies in this regard, implementing more studies in various fields is proposed for future works.

\section{Referenceses:}

[1]Montgomery DC, Peck EA. Translated by: Razavi Parisei SA. Statistics: Introduction to Linear Regression Analysis. 1st ed. Shahid Bahonar University of Kerman Publications, 1953; PP: 471-485, 501-503 (Persian)

[2]Johnson RA, Wichern DW. Translated by: Niroomand HA. Statistics: Applied Multivariate Statistical Analysis. 2nd ed. Mashhad Ferdowsi University Press, 1942; PP: 431-432 (Persian)

[3]McIntosh A R, Lobaugh NJ. Partial least squares analysis of neuroimaging data: applications and advances. NeuroImage 2004; 23: S250-S263.

[4]Sadeghi Bazargani H. Epidemiology and Statistical Modeling in Burn Injuries. Thesis submitted for epidemiology PhD degree, Karolinska Institutet, Sweden. 2010.

[5]Majidi Parsa T. Partial least squares regression and its applications. Thesis submitted for Statistics Msc degree, Islamic Azad University Tehran North Branch, 2008.

[6]Charles H, Disya R. Ultrasound and cerebral vascular disease. In: Toole JF, editor. Cerebrovascular disease, 5th ed. Philadelphia, Lippincott Williams \& Wilkins, 1998; PP: 83-128.

[7]Abdi H. Partial least squares regression and projection on latent structure regression (PLS Regression), Wiley \& Sons, Inc. WIREs Comp Stat. 2010,

[8]Gabrielsson J, Jonsson H, Airiau C, Schmidt B, Escott R, Trygg J. The OPLS methodology for analysis of multi-block batch process data. J Chemometrics 2006; 20: 362-369.

Internal repeat ${ }^{4}$ 
[9]Gabrielssona J, Jonsson H, Airiaub C, Schmidtb B, Escottb R, Trygg J. OPLS methodology for analysis of pre-processing effects on spectroscopic data. Chemometrics and Intelligent Laboratory Systems 2006; 84: 153-158.

[10]Jalali-Heravi M, Ebrahimi-Najafabadi H, Khodabandehloo A. Use of Kernel Orthogonal Projection to Latent Structure in Modeling of Retention Indices of Pesticides. QSAR Comb Sci 2009; 28,: 1432-1441.

[11]Eriksson L, Johansson E, Wold N, Trygg J, Wikstrom C, Wold S. Multi- and Megavariate data analysis: Advanced applications and method extensions. 1st ed. Umea, Umetrics AB, 2006; PP:

[12]Trygg J, Wold S. Orthogonal projections to latent structures (O-PLS). J Chemometrics 2002; 16: $119-128$.

[13]Bylesjo M, Rantalainen M, Cloarec O, Nicholson JK, Holmes E, Trygg J. OPLS discriminant analysis: Combining the strengths of PLSDA and SIMCA classification. $J$ Chemometr 2006; 20(810): 341-351.

[14]Sadeghi-Bazargani H, Bangdiwala SI, Mohammad K, Maghsoudi H, Mohammadi R. Compared application of the new OPLS-DA statistical model versus partial least squares regression to manage large numbers of variables in an injury case-control study. Scientific Research and Essays 2011; 6(20): 4369-4377.

[15]Charles H, Disya R. Ultrasound and cerebral vascular disease. In: Toole JF, editor. Cerebrovascular disease. 5th ed. Philadelphia, Lippincott Williams \& Wilkins, 1998; PP: 83-128.

[16]Rajamany K, Gorman M. Transcranial doppler in stroke. Biomed Pharamacother 2001; 55: 24757.

[17]Wechsler LR. Cerebrovascular diseases. In: Babikian VL, Wechsler LR, editors. Transcranial doppler ultrasonography. 2nd ed. USA, Butterworth-Heinemann, 1999; PP: 91-108.

[18]Mehdizadeh Esfanjani R. Comparison of PLS and OPLS methods in controlling the variety of variables. Example: A Sample in TCD. Thesis submitted for Statistics Msc degree, Islamic Azad University Tehran North Branch, 2011.

[19]Dohoo IR, Ducrot C, Fourichon C, Donald A, Hurnik D. An overview of techniques for dealing with large numbers of independent variables in epidemiologic studies. Prev Vet Med 1997; 29(3): 221-239.

[20]Wold S, Antti H, Lindgren F, Ohman J. Orthogonal signal correction of near-infrared spectra. Chemometrics Intell Lab Syst 1998; 44: 175-185.

[21] M. Bagheri, M. Valipour, V. Amin, The Bankruptcy Prediction in Tehran share holding using Neural Network and it's Comparison with Logistic Regression,5(3),pp-219- 228.(2012).

[22] A. Arjmandzadeh, S. Effati, Interval Support Vector Machine in Regression Analysis, 2(3), pp 565- 571 (2011). 
K.F. Vajargah, R. Mehdizadeh, H. Sadeghi-Bazargani / J. Math. Computer Sci. 8 (2014), 411-422

[23] A.H. Hadjahmadi, T. J. Askari, A Decision Support System for Parkinson's Disease Diagnosis using Classification and Regression Tree, 4(2) pp-257 - 263,(2012). 